\title{
Regularization of inverse photomask synthesis to enhance manufacturability
}

\author{
Ningning Jia ${ }^{a}$, Alfred K. Wong, and Edmund Y. Lam ${ }^{a}$ \\ ${ }^{a}$ Imaging Systems Laboratory, Department of Electrical and Electronic Engineering, \\ The University of Hong Kong, Pokfulam Road, Hong Kong
}

\begin{abstract}
Mask manufacturability has been considered as a major issue in the adoption of inverse lithography (IL) in practice. With smaller technology nodes, IL distorts the mask pattern more aggressively. The distorted mask often contains curvilinear contour and irregular shapes, which cast a heavy computation burden on segmentation and data preparation. Total variation (TV) has been used for regularization in previous work, but it is not very effective in regulating the mask shape to be rectangular. In this paper, we apply TV regularization not only on the mask image but also on the mask edges, which forces the curves of edges to be more vertical or horizontal, because they give smaller TV values. Except for rectilinearity, a group of geometrical specifications of the mask pattern set by mask manufacture rule control (MRC) is also important for mask manufacturability. To prevent these characteristics from appearing, we also propose an intervention scheme into the optimization framework.
\end{abstract}

Keywords: Inverse lithography, mask manufacturability, regularization on edge, intervention scheme, MRC

\section{INTRODUCTION}

Inverse lithography (IL) formulates photomask design as an inverse problem, and derives optimal masks as solutions. Since IL is not constrained by the topology of the target design, it is able to generate unintuitive solutions, bearing little resemblance to the original one, while delivering superior on-wafer performance. ${ }^{1}$ However, IL brings irregular shapes and scattered small holes, resulting in masks that are often too complicated to manufacture. ${ }^{2-4}$ Moreover, the curvilinear feature contours result in a large number of fracture rectangles during segmentation. The cost of manufacturing such masks therefore is tremendously increased. Due to the lack of a mathematical description on mask regularization providing suitable control on issues such as mask manufacturability in the optimization process, the application of IL algorithms is still limited. ${ }^{2}$

Some efforts on regularization algorithms have been made to control the mask shape. Poonawala and Milanfar ${ }^{5}$ add the total variation (TV) regularization term to suppress small features. Ma and Arce ${ }^{6}$ use wavelet penalty to remove details on the mask. Besides, Shen and Yu clean up the mask image by optimizing a set of DCT low frequency coefficients. ${ }^{7}$ However, there has not been any explicit control on the contour shape to force the curvilinear edge to be more rectilinear. In this paper, we propose a regularization to mandate more regular mask shapes by applying TV regularization on image edges within an iterative optimization framework.

The regulated mask image, however, may still have some geometrical characteristics that are undesirable for mask manufacturing. Mask manufacture rule check (MRC) sets more specific constraints on the mask geometry. These concerns are more detailed and difficult to address all within one or more regularization terms. Therefore, we introduce some interventions into the optimization process to achieve better mask shapes.

\section{FRAMEWORK AND REGULARIZATION}

This paper enhances the iterative optimization framework with defocus variation. ${ }^{4}$ Masks are represented by pixel-based 2-D images, and gradient descent is applied to the iterative optimization. ${ }^{5}$ Before presenting the mathematical description, we first explain system models.

In this paper, we conduct our study on binary masks in a coherent imaging system for simplification. The algorithm can be further expanded to more sophisticated models such as phase shift masks in partially coherent imaging. We use $M, I, \hat{I}$ to represent the mask, the on-wafer pattern and the target pattern respectively.

Lithography Asia 2009, edited by Alek C. Chen, Woo-Sung Han, Burn J. Lin, Anthony Yen,

Proc. of SPIE Vol. 7520, 75200E · @ 2009 SPIE · CCC code: 0277-786X/09/\$18 · doi: 10.1117/12.837512

Proc. of SPIE Vol. $752075200 E-1$ 
For binary masks, the pixel values are constrained to $\{0,1\}$, and the optical transfer function (OTF) in the frequency domain, denoted by $\hat{H}(f, g)$, is given by ${ }^{8,9}$

$$
\hat{H}(f, g)=\left\{\begin{array}{ll}
1, & \sqrt{f^{2}+g^{2}} \leq \frac{\mathrm{NA}}{\lambda} \\
0, & \sqrt{f^{2}+g^{2}}>\frac{\mathrm{NA}}{\lambda}
\end{array},\right.
$$

where NA is the numerical aperture, and $\lambda$ represents the wavelength.

Taken the defocus variation as a random variable, denoted by $\beta$, the OTF is modified by adding an exponential phase term, ${ }^{10}$ which, in spatial domain, is given by

$$
H(x, y ; \beta)=\mathcal{F}^{-1}\left\{\hat{H}(f, g) \mathrm{e}^{-j \pi\left(f^{2}+g^{2}\right) \beta}\right\},
$$

where $\mathcal{F}^{-1}$ represents inverse Fourier Transform.

The aerial image is then calculated by convolving the mask transmittance with the OTF defined in Equation 2. We also approximate the resist effect by a "sigmoid" function, where $a$ is the contrast adjustment parameter, and $t_{\mathrm{r}}$ defines the threshold. The on-wafer pattern $I$ is calculated by

$$
I=\frac{1}{1+\mathrm{e}^{-a\left(|M(x, y) * H(x, y ; \beta)|^{2}-t_{\mathrm{r}}\right)}} .
$$

We calculate the optimal mask by minimizing the mean square error (MSE) of the output image and the target pattern, formulated by

$$
M_{\mathrm{opt}}=\underset{M(x, y) \in\{0,1\}}{\arg \min } \mathcal{E}_{\beta}\left\{\sum_{x, y}[I(x, y)-\hat{I}(x, y)]^{2}\right\},
$$

where $\mathcal{E}_{\beta}$ denotes expectation over random variable $\beta$.

Equation 1 stipulates that $M(x, y) \in\{0,1\}$, which cause the above a combinatorial optimization problem. We adopt a common approach to relax the constraint $M(x, y) \in\{0,1\}$ to $0 \leq M(x, y) \leq 1$. That makes the mask $M$ in the optimization a gray-level image. The final optimized mask $M_{\mathrm{opt}}$ is a re-binarized image.

An optimization problem without manufacturability constraints is described by Equation 1. Since TV can repress small features through minimizing the image curve length, the optimization is recast as a regularized problem, given by

$$
M_{\mathrm{opt}}=\underset{0 \leq M(x, y) \leq 1}{\arg \min }\left\{C(M(x, y))+\mu R_{\mathrm{TV}}(M(x, y))\right\} .
$$

In Equation 2, $C(M(x, y))$ represents the objective function in Equation 1, $\mu$ is the Lagrange multiplier to define the aggressiveness of the regularization, and $R_{\mathrm{TV}}(M(x, y))$ is the TV regularization term, given by

$$
R_{\mathrm{TV}}(M(x, y))=\left\|Q_{\mathrm{x}}|M(x, y)-\hat{I}(x, y)|\right\|_{1}+\left\|Q_{\mathrm{y}}|M(x, y)-\hat{I}(x, y)|\right\|_{1},
$$

where $Q_{\mathrm{x}}$ and $Q_{\mathrm{y}}$ represent the first-order difference along $x$-axis and $y$-axis respectively, and $\|\cdot\|_{1}$ denotes the $\ell_{1}$ norm. Here the regularization is applied on the difference between the mask and the target pattern, since the mask is the distorted version of the target. This regularization is meaningless $\left(R_{\mathrm{TV}}(M(x, y))=0\right)$ when the mask is the exact copy of the target pattern $\hat{I}$ or $1-\hat{I}$. The former means no distortion is occurred, while the latter case usually declares the failure of divergence. In both cases the manufacturability is not a concern any more.

Although total variation is able to suppress isolated holes and serrated edges, it is not very effective to regulate mask shape to be rectangular, but allow diagonal lines to exist. Along the diagonal jagged edge, every single small step (jag) stands for a vertex of the polygon. Therefore, we apply TV regularization on the edge 


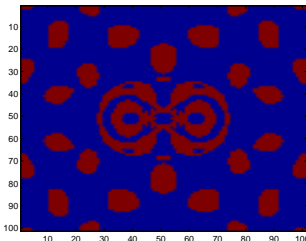

(a) The optimized mask without regularization on shape

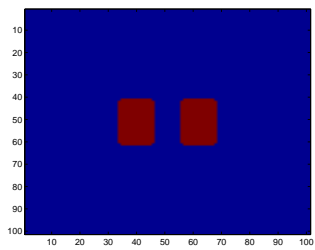

(e) On-wafer pattern of mask (a) on nominal focus conditions, pixel error $=8$

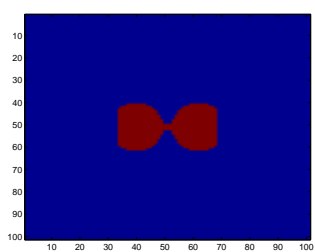

(i) On-wafer pattern of mask (a), $290 \mathrm{~nm}$ defocus, pixel error $=107$

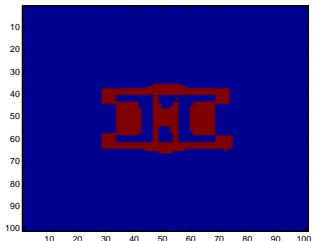

(b) The optimized mask with $\lambda_{\mathrm{TV}}=0.2$

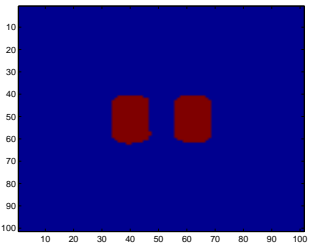

(f) On-wafer pattern of mask (b) on nominal focus conditions, pixel error $=27$

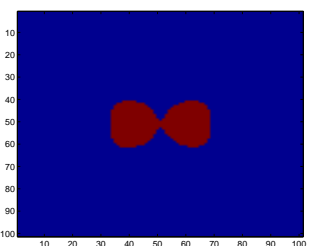

(j) On-wafer pattern of mask (b), $290 \mathrm{~nm}$ defocus, pixel error $=140$

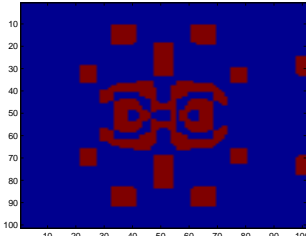

(c) The optimized mask with $\lambda_{\mathrm{TV}}=0.015$

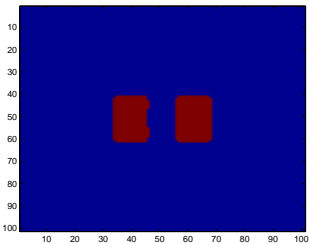

(g) On-wafer pattern of mask (c) on nominal focus conditions, pixel error $=18$

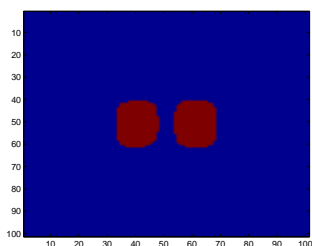

(k) On-wafer pattern of mask (c), $290 \mathrm{~nm}$ defocus, pixel error $=80$

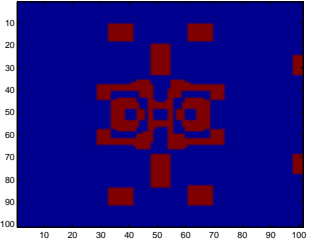

(d) The optimized mask with $\lambda_{\mathrm{TV}}=0.015$ and $\lambda_{\text {edge }}=$ 0.005

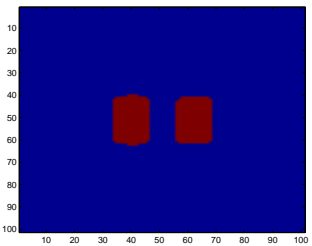

(h) On-wafer pattern of mask (d) on nominal focus conditions, pixel error $=18$

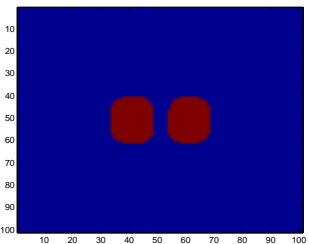

(1) On-wafer pattern of mask (d), $290 \mathrm{~nm}$ defocus, pixel error $=96$

Figure 1. Experimental results of pattern \#1.

of the mask image. The regularization on edge forces the curves of edges to be more vertical or horizontal by minimizing the number of vertices. The proposed regularization term is given by

$$
\begin{aligned}
R_{\text {edge }}(M(x, y)) & =\left\|Q_{\mathrm{x}} E_{\mathrm{y}}\right\|_{1}+\left\|Q_{\mathrm{y}} E_{\mathrm{x}}\right\|_{1} \\
& =\left\|Q_{\mathrm{y}} Q_{\mathrm{x}}|M(x, y)-\hat{I}(x, y)|\right\|_{1}+\left\|Q_{\mathrm{x}} Q_{\mathrm{y}}|M(x, y)-\hat{I}(x, y)|\right\|_{1},
\end{aligned}
$$

where $E_{\mathrm{x}}$ and $E_{\mathrm{y}}$ are edge images calculated along $x$-axis and $y$-axis respectively.

With the proposed regularization and the TV regularization, the regularized optimization problem is described as

$$
M_{\mathrm{opt}}=\underset{M(x, y) \in\{0,1\}}{\arg \min }\left\{C(M(x, y))+\mu_{\mathrm{TV}} R_{\mathrm{TV}}(M(x, y))+\mu_{\text {edge }} R_{\text {edge }}(M(x, y))\right\} .
$$

Some results by solving the problem in Equation 3 are shown in Figure 1, in which (a), (b), (c) and (d) represent optimized masks without shape regularization, with aggressive TV regularization, mild TV regularization and mild TV regularization with the proposed regularization together respectively. Aggressive TV regularization deteriorates on-wafer pattern fidelity at both infocus and defocus, while mild TV delivers better on-wafer patterns, but with more steps on its edge. Image (d) is optimized with TV both on the image and its edge. The mask contour becomes more rectilinear, and the printed layout suffers acceptable fidelity loss at the same time.

Notice that TV regularization is applied only on the changes $|M(x, y)-\hat{I}(x, y)|{ }^{5}$ It tends to keep the topology of the original design, while regulating the shapes of assist features. But it also, to some extent, limits 
the possible changes on main features. So we apply the TV regularization on the whole image to allow more possible distortions on the mask.

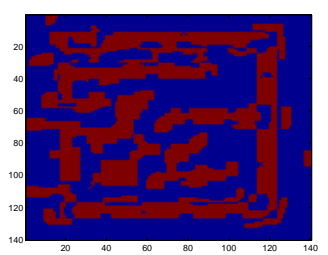

(a) The optimized mask with $\lambda_{\mathrm{TV}}=0.05$ on $|M-\hat{I}|$

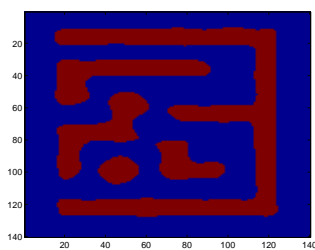

(e) On-wafer pattern of mask (a) on nominal focus conditions, pixel error $=424$

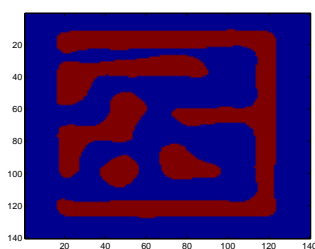

(i) On-wafer pattern of mask (a), $320 \mathrm{~nm}$ defocus, pixel error $=926$

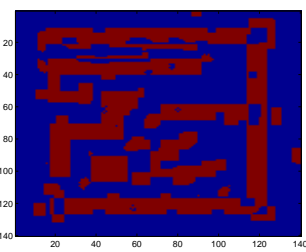

(b) The optimized mask with $\lambda_{\mathrm{TV}}=0.1$ on $|M-\hat{I}|$

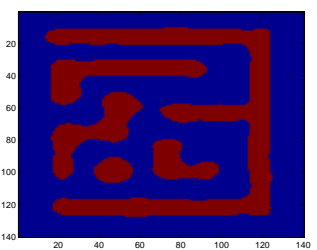

(f) On-wafer pattern of mask (b) on nominal focus conditions, pixel error $=495$

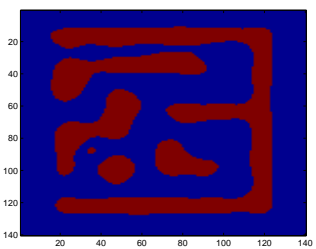

(j) On-wafer pattern of mask (b), $320 \mathrm{~nm}$ defocus, pixe error $=955$

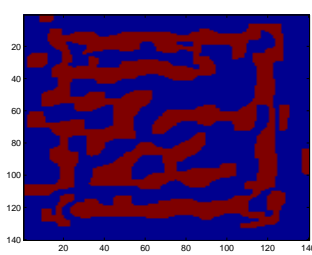

(c) The optimized mask with $\lambda_{\mathrm{TV}}=0.05$ on $M$

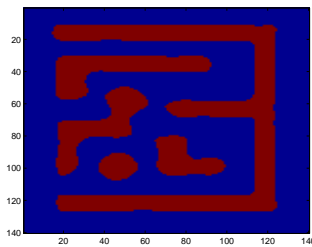

(g) On-wafer pattern of mask (c) on nominal focus conditions, pixel error $=349$

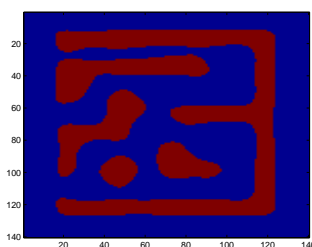

(k) On-wafer pattern of mask (c), $320 \mathrm{~nm}$ defocus, pixel error $=882$

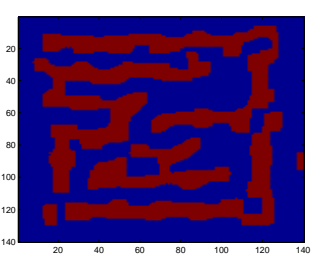

(d) The optimized mask with $\lambda_{\mathrm{TV}}=0.1$ on $M$

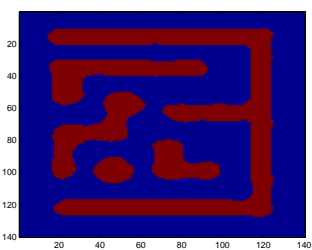

(h) On-wafer pattern of mask (d) on nominal focus conditions, pixel error $=391$

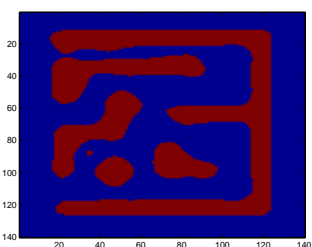

(1) On-wafer pattern of mask (d), $320 \mathrm{~nm}$ defocus, pixel error $=832$

Figure 2. Comparison of results by applying TV on the changes with TV on the whole image of pattern \#2.

In Figure 2, (a) and (b) are the optimized masks with TV regularization on the changes, while (c) and (d) are the optimized masks with TV on the whole mask images. Adding regularization only on the changes keeps the main features closer to their original shapes, and the serif-like assist features and some thin bars between two main features become more rectangular as the regularization gets more aggressive. However, some small holes still scatter inside the main features, as shown in (a) and (b). Instead, images with TV regularization on the whole mask images, like (c) and (d), show more feasibility of the pre-distortion and are also cleaner. Besides, with more freedom on pattern pre-distortion, (c) and (d) deliver on-wafer patterns with less fidelity loss under both nominal and defocus conditions.

Comparing (a) and (b) with (c) and (d) in Figure 2, it is obvious that the pattern curve of the latter two are much more curvilinear. Hence we apply the proposed regularization together with TV on the whole mask image. Figure 3 shows that the contours of these two masks optimized by adding the proposed regularization are more rectilinear.

\section{INTERVENTION SCHEME}

Notice all optimized masks above have some features like holes, thin bars and spaces, and closer corners. These features are undesirable in manufacturing. Mask Manufacture Rule Check (MRC) defines some rules on geometrical characteristics of mask features. ${ }^{11,12}$ The mask data of some features may not be properly resolved 


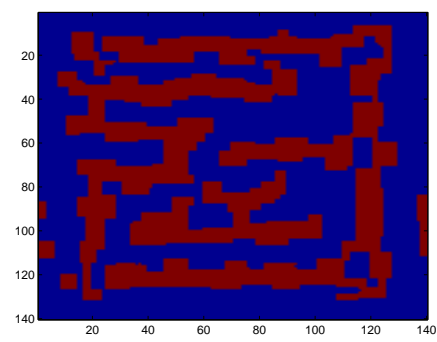

(a) The optimized mask with $\lambda_{\mathrm{TV}}=0.05$ and $\lambda_{\mathrm{Edge}}=0.025$ on $M$

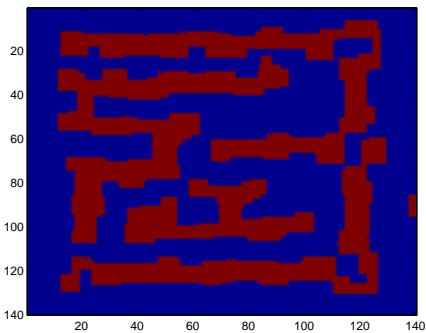

(d) The optimized mask with $\lambda_{\mathrm{TV}}=0.1$ and $\lambda_{\text {Edge }}=0.012$ on $M$

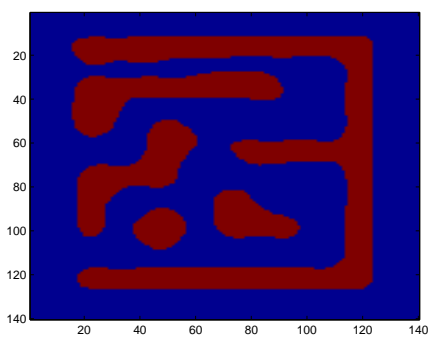

(c) On-wafer pattern of mask (b), $320 \mathrm{~nm}$ defocus, pixel error $=798$ inal focu 393
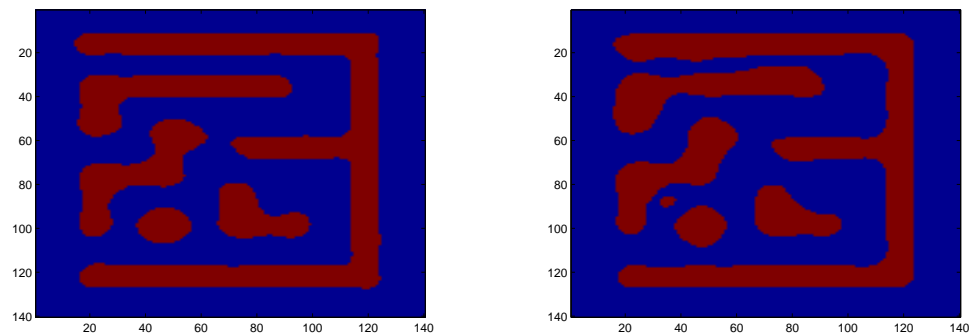

(e) On-wafer pattern of mask (f) on nominal focus conditions, pixel error $=359$ (f) On-wafer pattern of mask (f), $320 \mathrm{~nm}$ defocus, pixel error $=820$

Figure 3. Experimental results of pattern \#2 with TV both on the whole image and the edge.

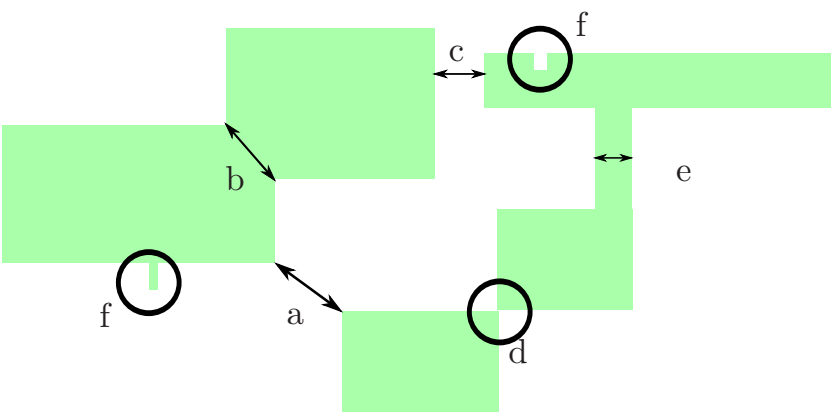

Figure 4. MRC sensitive regions.

by the mask writing tools, leading up to failure on mask manufacturing. Some features of concern in MRC are given in Figure 4, in which

- a : Distance between two vertices.

- b : Width of corner connections.

- c : Width of spaces.

- $\mathrm{d}$ : Kissing corners.

- e : Width of CD.

- f : Notches. 
MRC sets constraints on those patterns to reduce mask manufacturing cost. IL is very likely to bring some patterns within MRC's concern, in other words, MRC rules violation. Therefore, besides the regularization on edge to make the mask boundary more rectilinear, we also insert intervention schemes into the optimization process, leading to more acceptable results for manufacturing. We classify our interested patterns into 3 categories in the scheme:

\section{Corners.}

2. Thin connections.

\section{Holes.}

We propose a "remedy" for each category, and each will be deliberately inserted after every certain iteration steps. Sometimes the correction traps the optimization in a worse local minimum. Therefore we need to assess if a remedy is really useful, clearing MRC violation regions at the expense of acceptable fidelity loss in the printed pattern. If the correction is not worthwhile, like resulting in serious on-wafer pattern deformation, we will abandon the changes. Figure 5 draws the whole picture of the scheme.

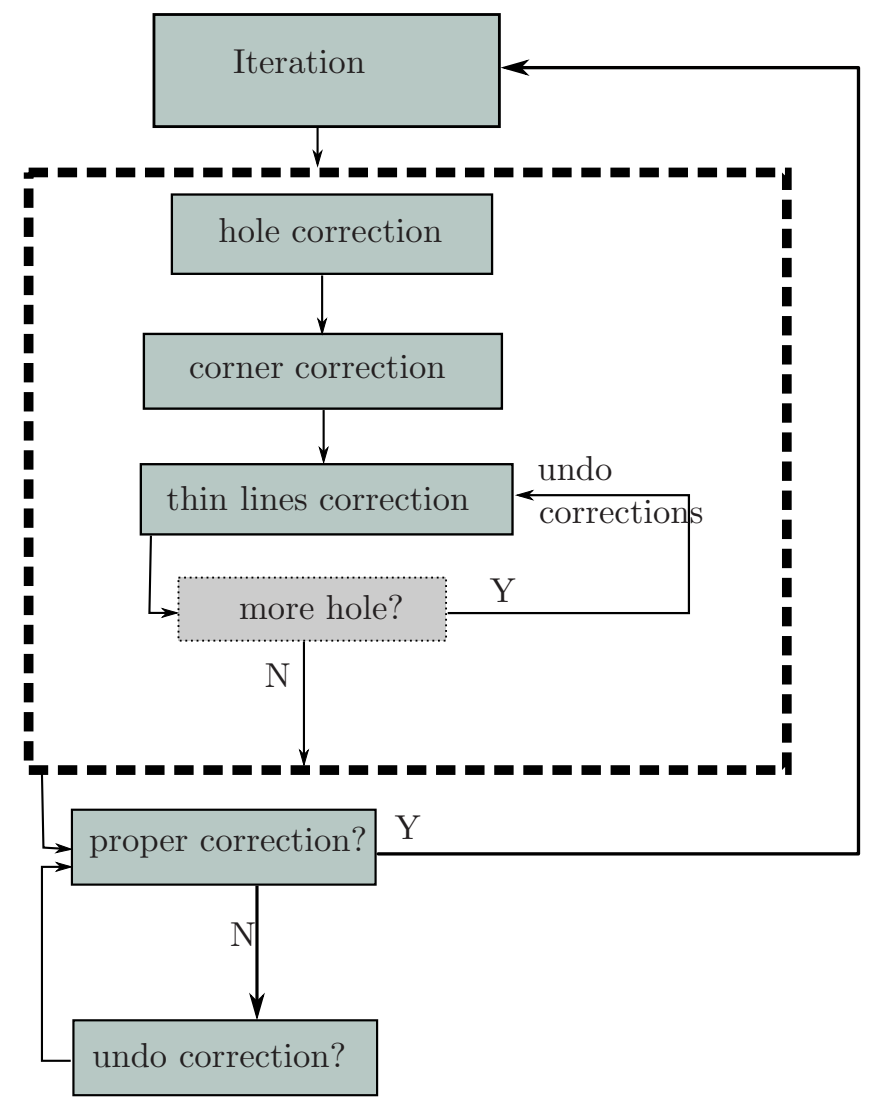

Figure 5. Intervention scheme.

In the following we give details of the three remedies.

1. Remedy for holes in the mask patterns.

Since in this paper we focus our study on binary masks, the image pixel value 1 denotes transparent regions and 0 represents opaque regions. Hole is defined as opaque regions surrounded by transparent regions. Some small holes (or dots) appear in the middle of a pattern, which will not cause much fidelity loss if we just fill in it. Some holes are due to serifs and thin or unstable connections. We aim to break 
holes because holes bring more fractured rectangles, and sometimes the presence of holes is accompanied by MRC violations such as thin connections and closer corners. We choose a simple strategy that we break every holes detected with its area larger than a certain threshold.

Let us see the surrounding 1 region as walls. We choose the thinnest part to break. First we locate a hole and the curve edges of its surrounding pattern, and then find the nearest points on the edge curve to the hole by calculating a distance map, illustrated in Figure 6. In this map the blue polygon is a hole detected on the mask, and the boundary of its surroundings is drawn by different colors. Each pixel value of the boundary is the Euclidean distance from the pixel to the hole. Therefore we can identify the thinnest part of the hole's surroundings in this map.

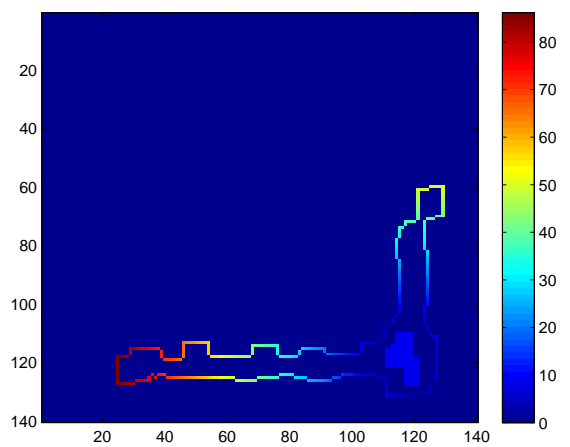

Figure 6. Distance map.

There might be more than one set of points (4-connected sets) with the minimum distance value $r_{\min }$. The set with a maximum area is located and a square window with its edge length $d$ centered at each set defines the opening area. Here $d$ is the nearest integers greater than or equal to $r_{\min }$. The window shape and its size can be defined according to specific requirements. Here we try to break the hole with as small changes of the original result as possible to avoid over-correction. In this step the hole-breaking may create closer corners, which can be fixed in the next step. In Figure 7, (a) is the original mask, and (b) is its hole-breaking result. Notice the hole detection and distance map are calculated based on the binary image, but corrections are made in gray-level masks.

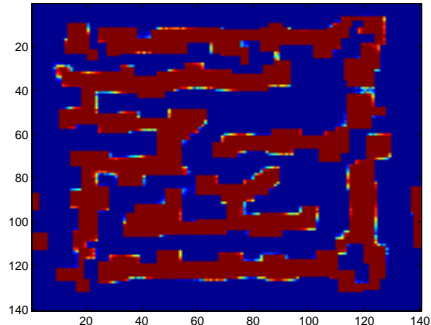

(a)

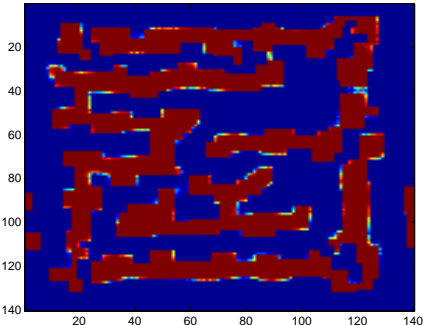

(b)

Figure 7. Hole breaking.

2. Remedy for closer corners.

In this step, we follow a routine of detection, classification and correction. A checkerboard filter, which is illustrated in Figure 8 is used to detect suspicious closer corners with small corner-corner distances. Every small grid stands for one pixel. Grids with gray color have pixel value 1, and white grids are pixels valued 
-1. Detected regions with strong response are marked as illustrated in Figure 9 (a). The red pixels point suspicious closer-corner regions.

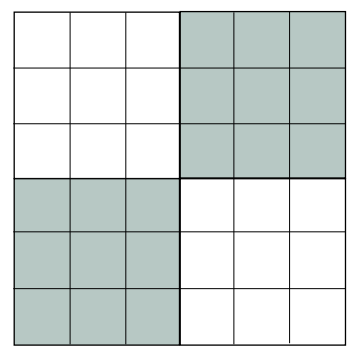

Figure 8. Checkerboard-like filter.

After detection, we narrow down our focus on every suspect's neighborhood regions, marked by small windows, as shown in Figure 9 (b).

The located suspicious corners are divided into two groups:

- Connecting corners, illustrated by Figure 4 (b) and (d).

- Closer corners, illustrated by Figure 4 (a).

For the first case, the pixels' value of their neighborhoods defined by small windows are set to 1 , in order to thicken the connection. If any connection made by correction creates extra holes, the window area is cleared to be 0 .

For the second case, the window area is set to be 0 to enlarge the distance of the two vertices.

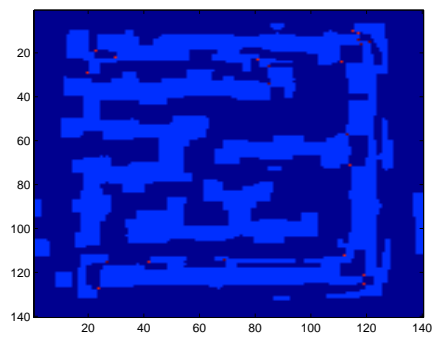

(a)

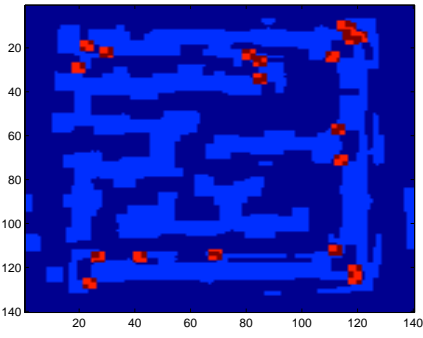

(b)

Figure 9. Corner detection and windowing.

3. Remedy for notches, thin connections or spaces.

Morphological smoothing on gray-level images can eliminate or reduce small size bright and dark details by applying opening operators first, then closing operators. ${ }^{13}$ Such properties can be used to clear thin connections and bridge across narrow spaces. However, connecting can also make holes. Therefore a recheck mechanism is required to avoid creating previous undesired patterns. Assuming all holes are successfully broken in the first step, we check if new holes appear again. The correction making new holes must adhere to the hole, so we locate the hole and check any correction adhering to it, and identify it as unnecessary correction. Once it is identified, this thin connection and certain area of its neighborhood are set to 0 to avoid narrow spaces.

We insert intervention into optimization iterations to change the path leading to a minimum point. Sometimes it over-corrects and will make the optimization trapped in a worse local minimum. In such cases some 
fatal pattern loss as pattern splitting, small pattern missing, and bridging between two adjacent features can be observed in the output images. Thus a check module is necessary to ensure the convergence to a local minimum. To achieve this, first we detect fatal failures on the output image after certain iteration steps (100 steps in this paper). If any failure is detected, we undo previous corrections.

\section{RESULTS}

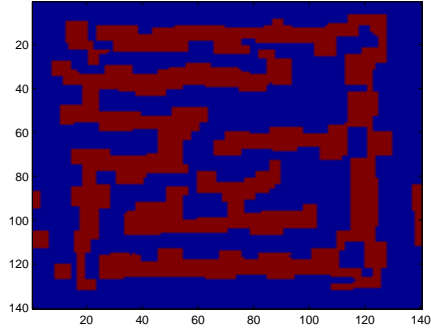

(a) Mask optimized without intervention, with $\lambda_{\mathrm{TV}}=0.05$ and $\lambda_{\mathrm{Edge}}=0.025$

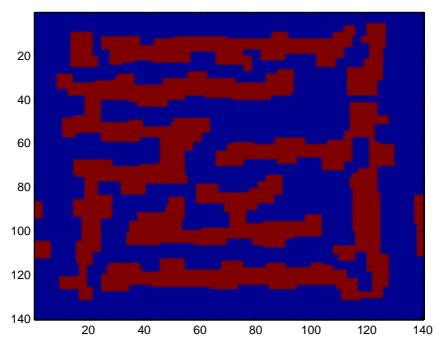

(d) Mask optimized with intervention

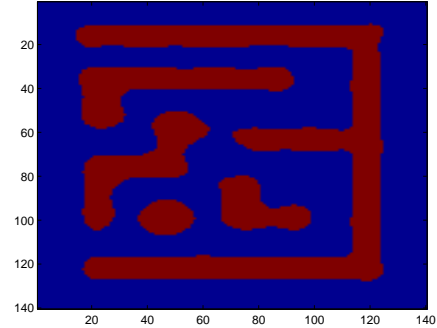

(b) Output of (a), infocus, pixel error = 393

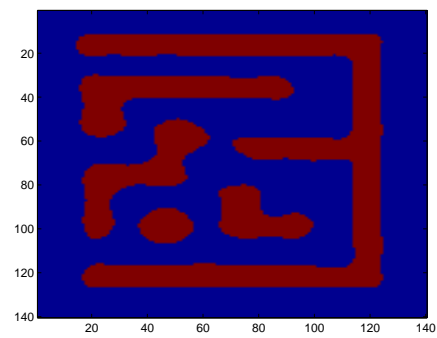

(e) Output of (d), infocus, pixel error $=$ 379

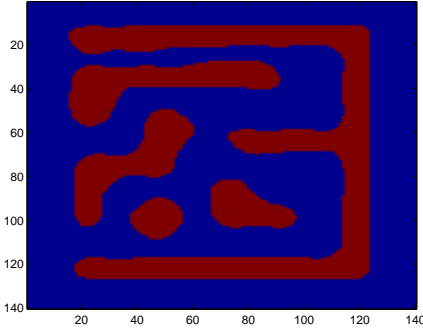

(c) Output of (a), defocus 320nm, pixel error $=798$

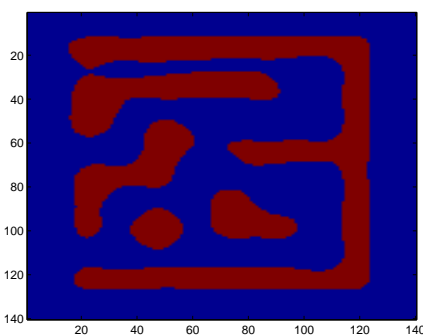

(f) Output of (d), defocus 320nm, pixel error $=862$

Figure 10. Results of pattern \#2 with mild shape regularization and intervention.

Figure 10 and Figure 11 show some optimized results with intervention under different degrees of shape regularization. The hole in Figure 10 (a) is broken in (d), and the distance of closer corners in Figure 10 (a) and Figure 11 (a) are increased in Figure 10 (d) and Figure 11 (d) respectively, and notches are gone. Notice in Figure 10 (d), hole breaking brings more steps, which result in more fractured rectangles. If the existence of the hole creates no MRC violations, the number and size of the fractured rectangles before and after hole breaking need to be evaluated to determine if the correction is necessary. This procedure can be a part of expansion of current intervention scheme.

Another concern of the intervention scheme is its effect on optimization convergence. Here the first intervention is inserted after 100 iterations, where the cost function has converged near some local minimum. We assume that local manipulation of pixels within a small range won't cause large scale fidelity loss in the output image. In the intervention scheme, corrections in each step are restricted in certain size of small windows. If the correction is large enough to cause fatal fidelity loss, it will be canceled. Moreover, the number of iteration loops between every two intervention operations is large enough to reach a local minimum again. Figure 12 draws the curves of cost function value along iteration steps of the two optimized masks in Figure 11. The $x$-axis denotes iteration numbers, and the $y$-axis is the value of the cost function. The blue curve represents iterations with intervention, while the magenta dashdot curve represents iterations without intervention. Intervention is inserted every 100 steps, where small blue glitches are observed at the 100th, 200th, and 300th steps. We could 


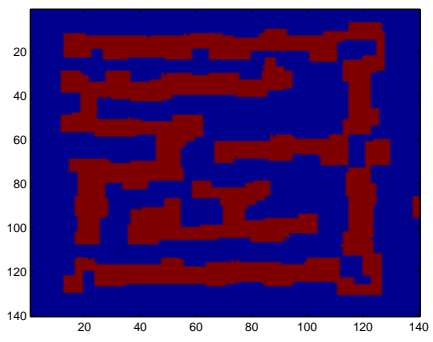

(a) Mask optimized without intervention, with $\lambda_{\mathrm{TV}}=0.1$ and $\lambda_{\mathrm{Edge}}=0.012$

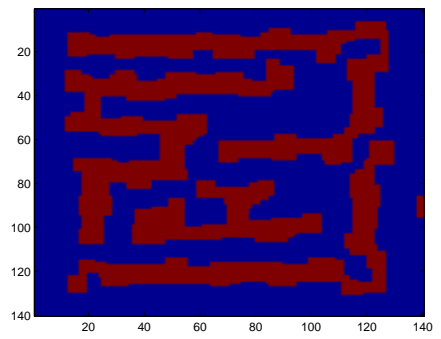

(d) Mask optimized with intervention

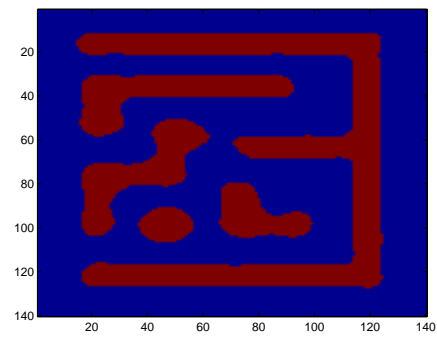

(b) Output of (a), infocus, pixel error $=$ 359

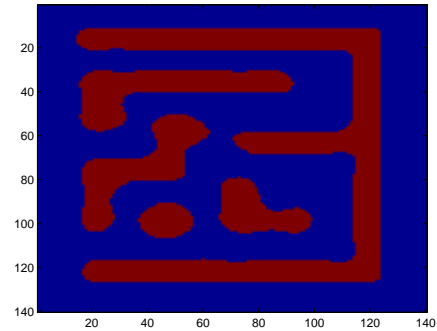

(e) Output of (d), infocus, pixel error = 371

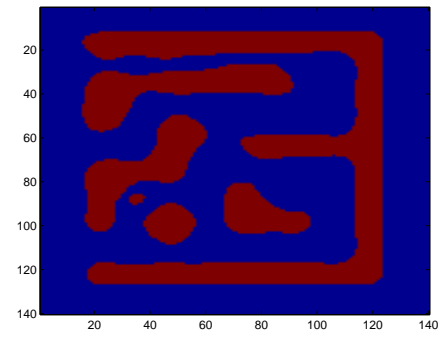

(c) Output of (a), defocus 320nm, pixel error $=820$

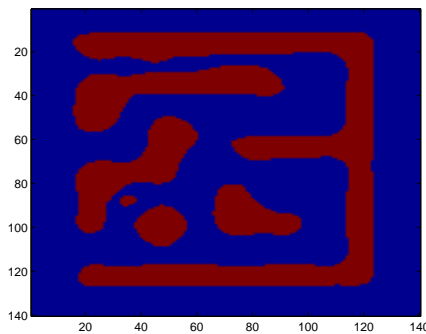

(f) Output of (d), defocus 320nm, pixel error $=875$

Figure 11. Results of pattern \#2 with aggressive shape regularization and intervention.

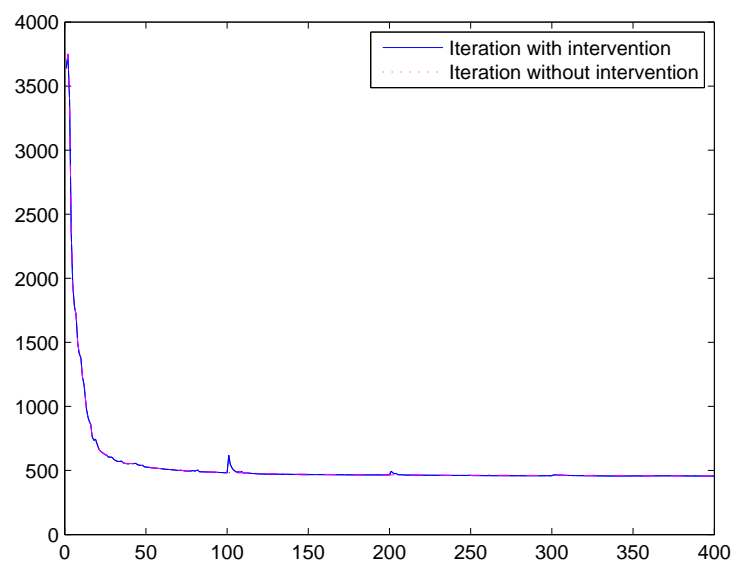

Figure 12. Steepest descent iterations with intervention.

see these two curves almost overlap with each other. Furthermore, the increments of cost function value brought by local pixel manipulation decreases as the intervention times increases.

\section{CONCLUSION}

This paper introduces a modified total variation regularization to increase mask manufacturability. TV regularization is applied not only in the whole mask image to suppress details and regulate shapes, but also in the edge of mask images to force the curve to be more vertical or horizontal. Therefore the contour of regulated images become more rectangular. We also propose an intervention scheme in the optimization process to break certain patterns that may violate MRC rules by deliberately inserting local corrections. Simulation results prove this is effective to avoid unwanted patterns to some extent. 


\section{ACKNOWLEDGMENTS}

This work was supported in part by the Research Grants Council of the Hong Kong Special Administrative Region, China under Projects HKU 7139/06E and 7134/08E.

\section{REFERENCES}

1. Y. Liu, D. Abrams, L. Pang, and A. Moore, "Inverse lithography technology principles in practice : Unintuitive patterns," in 25th Annual BACUS Symposium on Photomask Technology, J. T. Weed and P. M. Martin, eds., Proc. SPIE 5992, 2005.

2. E. Y. Lam and A. K. Wong, "Computation lithography: virtual reality and virtual virtuality," Optics Express 17, pp. 12259-12268, 2009.

3. X. Ma and G. R. Arce, "Binary mask optimization for inverse lithography with partially coherent illumination," in Lithography Asia 2008, A. C. Chen, B. Lin, and A. Yen, eds., Proc. SPIE 7140, 2008.

4. N. Jia, A. K. Wong, and E. Y. Lam, "Robust mask design with defocus variation using inverse synthesis," in Lithography Asia 2008, A. C. Chen, B. Lin, and A. Yen, eds., Proc. SPIE 7140, 2008.

5. A. Poonawala and P. Milanfar, "Mask design for optical microlithography - An inverse imaging problem," IEEE Trans. Image Process. 16, pp. 774-788, 2007.

6. X. Ma and G. R. Arce, "Generalized inverse lithography methods for phase-shifting mask design," in Optical Microlithography XX, D. G. Flagello, ed., Proc. SPIE 6520, 2007.

7. S. Shen, P. Yu, and D. Z. Pan, "Enhanced DCT2-based inverse mask synthesis with initial SRAF insertion," in Photomask Technology 2008, H. Kawahira and L. S. Zurbrick, eds., Proc. SPIE 7122, 2008.

8. A. K. Wong, Resolution Enhancement Techniques in Optical Lithography, SPIE, Washington, 2001.

9. J. W. Goodman, Introduction to Fourier Optics, Roberts \& Company, Greenwood Village, 2005.

10. S. Förster, H. Gross, F. Höller, L. Höring, and C. Zeiss, "Extended depth of focus as a process of pupil manipulation," in Optical Design and Engineering II, L. Mazuray and R. Wartmann, eds., Proc. SPIE 5962, p. 596202, 2005.

11. J. Strauba, D. Aguilara, P. Buckb, D. Dawkinsa, R.Gladhillb, S. Nolkeb, and J. Riddicka, "Advanced manufacturing rules check (MRC) for fully-automated assessment of complex reticle designs -part II," in Photomask Technology 2006, P. M. Martin and R. J. Naber, eds., Proc. SPIE 6349, 2006.

12. A. Balasinski, D. Coburn, and P. Buck, "Mask manufacturability improvement by MRC," in Photomask Technology 2007, R. J. Naber and H. Kawahira, eds., Proc. SPIE 6730, 2007.

13. R. C. Gonzalez and R. E. Woods, Digital Image Processing, Prentice Hall, Upper Saddle River, N.J., 2002. 